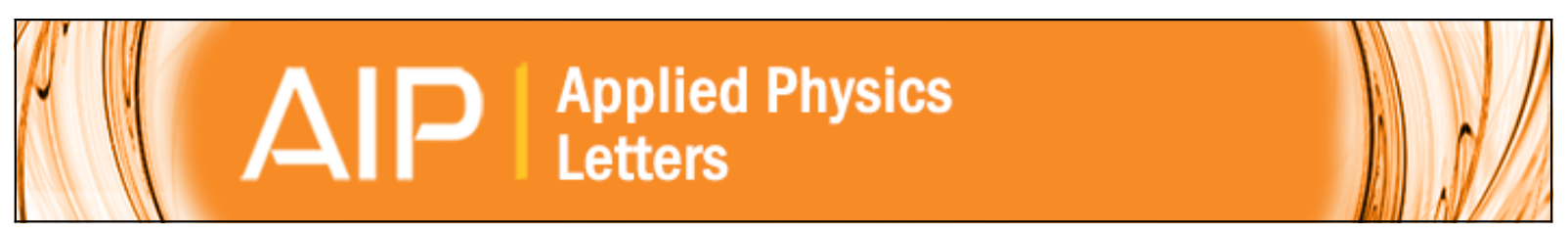

\title{
Carrier dynamics in InP nanopillar arrays fabricated by low-damage etching
}

S. Naureen, N. Shahid, A. Gustafsson, V. Liuolia, S. Marcinkeviius, and S. Anand

Citation: Applied Physics Letters 102, 212106 (2013); doi: 10.1063/1.4808447

View online: http://dx.doi.org/10.1063/1.4808447

View Table of Contents: http://scitation.aip.org/content/aip/journal/apl/102/21 ?ver=pdfcov

Published by the AIP Publishing

\section{Articles you may be interested in}

In situ electron-beam lithography of deterministic single-quantum-dot mesa-structures using low-temperature cathodoluminescence spectroscopy

Appl. Phys. Lett. 102, 251113 (2013); 10.1063/1.4812343

Gas chopping etching process for InP based nanostructures with high aspect ratios

J. Vac. Sci. Technol. B 30, 060601 (2012); 10.1116/1.4754293

Position-controlled [100] InP nanowire arrays

Appl. Phys. Lett. 100, 053107 (2012); 10.1063/1.3679136

Raman and photoluminescence characterization of focused ion beam patterned InGaN/GaN multi-quantum-wells nanopillar array

J. Appl. Phys. 110, 093111 (2011); 10.1063/1.3658866

Optical properties of InGaN/GaN nanopillars fabricated by postgrowth chemically assisted ion beam etching J. Appl. Phys. 107, 023522 (2010); 10.1063/1.3280032

\section{AlP Re-register for Table of Content Alerts}




\title{
Carrier dynamics in InP nanopillar arrays fabricated by low-damage etching
}

\author{
S. Naureen, ${ }^{1}$ N. Shahid, ${ }^{1}$ A. Gustafsson, ${ }^{2}$ V. Liuolia, ${ }^{1}$ S. Marcinkevičius, ${ }^{1}$ and S. Anand ${ }^{1, a)}$ \\ ${ }^{1}$ School of Information and Communication Technology, KTH Royal Institute of Technology, Electrum 229, \\ 16440 Kista, Sweden \\ ${ }^{2}$ Department of Solid State Physics, Lund University, Box 118, 22100 Lund, Sweden
}

(Received 9 February 2013; accepted 5 May 2013; published online 31 May 2013)

\begin{abstract}
We present a comprehensive characterization of the optical quality of InP nanopillars (NPs) fabricated by a top down approach using micro-photoluminescence ( $\mu$-PL), time-resolved PL, and cathodoluminescence (CL). A lattice matched InGaAs layer provided beneath the $1 \mu \mathrm{m}$ tall NPs functions as a "detector" in CL for monitoring carrier diffusion in InP NP. Carrier feeding to the InGaAs layer indicated by a double exponential PL decay is confirmed through CL mapping. Carrier lifetimes of over $1 \mathrm{~ns}$ and the appreciably long diffusion lengths (400-700 nm) in the InP NPs indicate very low surface damage making them attractive for optoelectronic applications. (C) 2013 AIP Publishing LLC. [http://dx.doi.org/10.1063/1.4808447]
\end{abstract}

One dimensional semiconductor nanostructures such as nano-wires (NWs) or nanopillars (NPs) have been the subject of intense research for the last two decades due to interesting fundamental physics and their potential to derive next generation electronics and optoelectronic devices. ${ }^{1-4} \mathrm{~A}$ broad spectrum of unique optical properties, such as nonlinearity, ${ }^{5,6}$ waveguiding, ${ }^{7,8}$ and nanoantenna effects ${ }^{9}$ make them attractive for compact nanophotonic devices. InP- and GaAsbased nanowires are prime candidates for optoelectronic devices including photovoltaics ${ }^{10}$ due to their direct bandgap and high carrier mobilities when compared with group IV semiconductors.

Surface states and crystallographic defects inadvertently affect recombination lifetimes and quantum efficiencies in nanowire systems including InP, CdS, GaAs, and GaAs/ GaAlAs. ${ }^{11-14}$ Generally, dangling bonds on nanowire or nanopillar surfaces trap carriers and are a channel of nonradiative recombination. However, surface recombination velocity also varies in different semiconductor materials. ${ }^{15}$ Due to a lower surface recombination velocity in $\operatorname{InP}\left(\sim 10^{3} \mathrm{~cm} / \mathrm{s}\right)$ compared to GaAs $\left(\sim 10^{6} \mathrm{~cm} / \mathrm{s}\right)$, charge carriers in InP are less affected by surface recombination. In epitaxially grown InP NWs, low radiative recombination lifetimes have been attributed to surface band bending, wurtzite-zinc blend poly-typism and crystallographic defects. ${ }^{16,17}$ Although tremendous efforts have been made in nanowire growth, improvement in structural morphology with precise control over their composition and dopant concentration is still required to get functional devices. ${ }^{18,19}$ NWs or NPs fabricated by top down approach ${ }^{20}$ using user designed epitaxial structures, grown by mature growth techniques can be an attractive alternative. However, top-down approaches invariably employ dry etching which can damage the surface, degrading optical properties. Thus, low damage processes are necessary. Further, it is essential to study carrier dynamics and transport properties such as recombination life-times and diffusion lengths in top down fabricated nanopillars (NPs) for device applications.

In this work, we focus on the optical properties of InP NP arrays fabricated by a combination of colloidal lithography

a)Electronic mail: anand@kth.se and dry etching. ${ }^{20}$ We have used micro-photoluminescence $(\mu$-PL) spectroscopy, time-resolved photoluminescence (TRPL), and cathodoluminescence (CL) to perform a comprehensive analysis of the optical quality of the InP NPs in terms of PL intensity, carrier lifetimes, and diffusion lengths.

The InP/InGaAs structure was grown by metal organic vapor phase epitaxy (MOVPE) on an $n+$ InP substrate. The sample structure consisted of $1 \mu \mathrm{m}$ thick InP on a $300 \mathrm{~nm}$ InGaAs (lattice matched to InP) layer. The epitaxially grown layers are nominally undoped. The NP arrays are fabricated using colloidal lithography and $\mathrm{Cl}_{2} / \mathrm{H}_{2} / \mathrm{CH}_{4}$ based inductively coupled plasma reactive ion etching (ICP-RIE) process. The sample has several areas, $1-2 \mathrm{~mm}^{2}$, with uniform NP arrays (Fig. 1(a)). Other regions of the sample have partial coverage. The InP pillars are $\sim 1 \mu \mathrm{m}$ tall with an average diameter of $200 \mathrm{~nm}$ (Fig. 1(b)), and have the InGaAs layer at their base. Detailed description of the fabrication process has been reported earlier. ${ }^{20}$

$\mu$-PL measurements were performed to characterize the PL of the fabricated InP NPs. The excitation spot size of $\sim 20 \mu \mathrm{m}$ was used to study PL intensity dependence on NP surface density/coverage. $\mathrm{An}^{+}{ }^{+} 514.5 \mathrm{~nm}$ laser was used for PL measurements due to its small penetration depth $(\sim 90 \mathrm{~nm})$ in InP. Moreover, the InGaAs layer effectively absorbs PL emission from the InP substrate. Figure 2 reveals very good luminescence properties of the NP arrays. The PL intensity for the uniform array is comparable to that of the as-grown layer under identical excitation conditions. Considering the low fill factor $(\sim 20 \%)$, this strongly indicates that the array geometry and shape of NPs contributes to enhanced extraction and absorption of light. The data of Fig. 2 show that the PL intensities qualitatively scale with the NP density. PL spectra of NPs measured at room temperature (Fig. 2) and $77 \mathrm{~K}$ (Fig. 2, inset) show line widths similar to that of the as grown reference sample. This clearly suggests the material quality of the NPs is comparable to the epilayer. However, independent measurements of carrier lifetime and diffusion length provide a direct evaluation of the optical quality of the fabricated NPs.

Time-resolved PL measurements were used to determine the carrier lifetimes at room temperature using a synchroscan streak camera with a time resolution of $2 \mathrm{ps}$. 

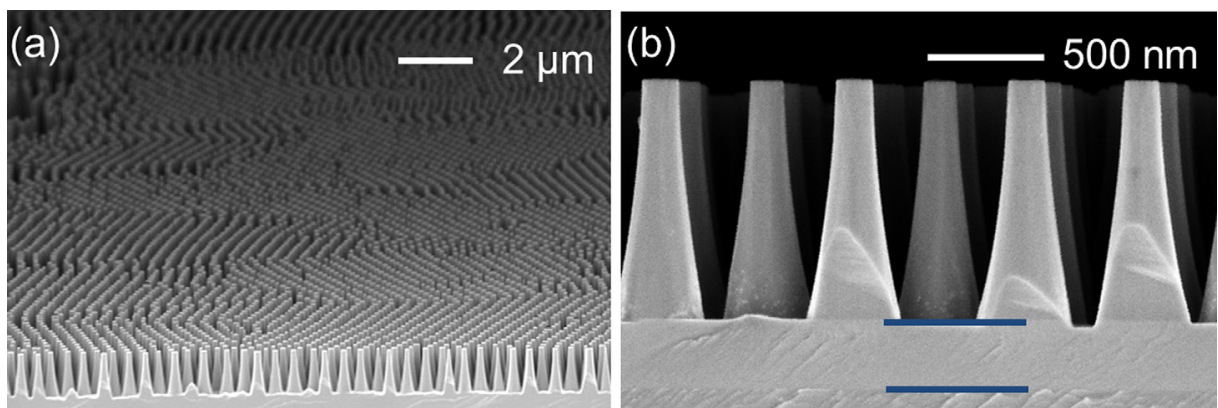

FIG. 1. SEM images of InP NPs fabricated by colloidal lithography and ICP-RIE etching (a) as-etched array (b) after removal of remaining colloidal silica particles. Material contrast for the InGaAs layer is visible in (b).
A tunable mode-locked Ti:sapphire laser (pulse temporal length $\sim 100 \mathrm{fs}$, repetition rate $76 \mathrm{MHz}$, wavelength $\sim 800 \mathrm{~nm}$ ) with a spot diameter of $20-25 \mu \mathrm{m}$ was used for excitation. The detected PL peak position was around $920 \mathrm{~nm}$ corresponding to the bandgap of epi-grown InP. As shown in Fig. 3, the measured PL decay curves show a double exponent behavior for the NPs on native substrate with the InGaAs layer. The PL decay for reference InP with the InGaAs layer shows a similar double exponent process (not shown here).

The measured TPRL decay curves were fitted using the following double exponential decay function:

$$
I(t)=I_{0}+I_{1} e^{-\left(t-t_{0}\right) / \tau_{1}}+I_{2} e^{-\left(t-t_{0}\right) / \tau_{2}},
$$

where $I(\mathrm{t})$ is the PL intensity at time $t$ and $I_{0}$ at $t_{0} . \tau_{1}$ and $\tau_{2}$ are the PL lifetimes, for the two processes 1 and 2 , respectively. $I_{1}$ and $I_{2}$ are constant coefficients. The fitted curves show very good agreement with measured TRPL data (Fig. 3). A fast decay time of $255 \mathrm{ps}$ and a slow one of $1.15 \mathrm{~ns}$ were obtained for NPs on the InGaAs layer. The short decay time $\tau_{1}$ is attributed to carrier feeding into the InGaAs layer, and $\tau_{2}$ to carrier recombination in the InP NPs. ${ }^{21,22}$ A large fraction of the photo-generated carriers that are not within the diffusion length from InGaAs layer recombine in the InP NP, and correspond to the longer decay time. To confirm the assumptions above, the carrier lifetimes of "free" InP NPs obtained by selectively etching the InGaAs layer (and transferred to a Si substrate) were measured.

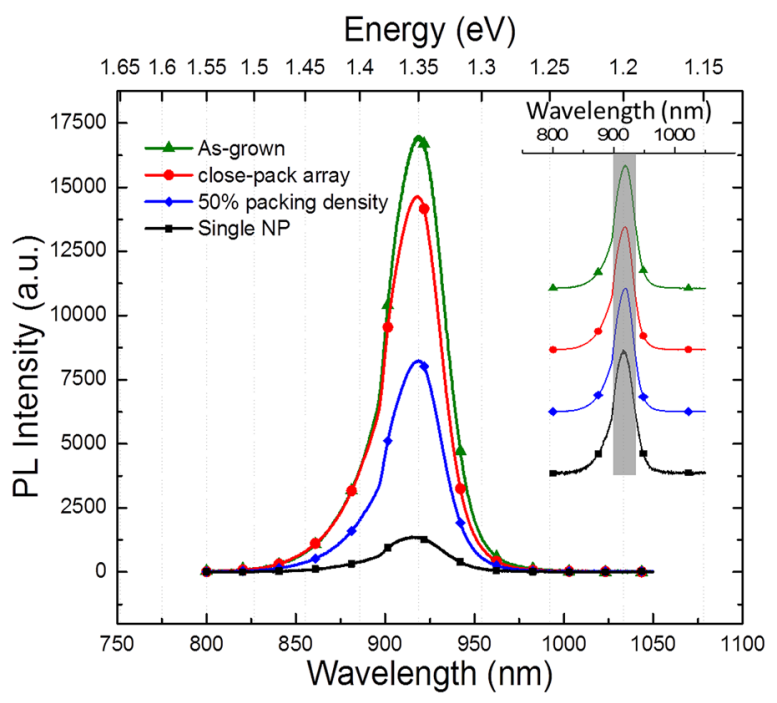

FIG. 2. Room temperature PL spectra of array and single NPs. Inset shows normalized PL spectra of the InP NPs and the reference InP wafer at $77 \mathrm{~K}$.
A single exponential decay (Fig. 3) with a lifetime of $1.15 \mathrm{~ns}$ is observed for the free NPs, indicating negligible surface damage. This is comparable to the PL-lifetime $(1.2 \mathrm{nS})$ obtained in the unprocessed reference sample. To estimate the effective diffusion length in the NPs, characteristic to the InP NP material, the appropriate lifetime is that obtained for the free InP NPs or the long lifetime $\left(\tau_{2}\right)$ obtained for the InP NP/InGaAs structure (Fig. 3). The effective diffusion length $\left(L_{D}\right)$ in the InP NPs corresponding to the measured lifetime of $1.15 \mathrm{~ns}$ is in the range of $500-775 \mathrm{~nm}$, which is calculated from the relationship $L D=\sqrt{D \tau}$, where the diffusion coefficients, $D=2.2-5 \mathrm{~cm}^{2} / \mathrm{s}$ are used. ${ }^{15,23}$

In order to confirm the draining of carriers to the underlying InGaAs and to determine the diffusion lengths in the InP segment of the NPs experimentally, CL measurements were performed using a dedicated SEM with a He cold stage. ${ }^{24}$ The acceleration voltage was $5 \mathrm{keV}$, the probe current $10-25 \mathrm{~Pa}$, and the sample temperature $8 \mathrm{~K}$. The NPs were investigated in side-view along the cleaved edge of the sample. The spatial resolution in CL imaging comes from the local excitation, where the detection has no spatial resolution. The spatial resolution is therefore limited by charge carrier diffusion in the sample under investigation and is used to determine ambipolar diffusion lengths from intensity profile. ${ }^{25,26}$ In the present structure, the lower bandgap material (InGaAs) can be used advantageously as a "detector" for the diffusing carriers in the InP NP. Fig. 4(a) shows a typical SEM image of an area with NPs together with monochromatic CL images in Fig. 4(b) for $\operatorname{InP}(\lambda=876 \mathrm{~nm})$ and in Fig. $4(\mathrm{c})$ for InGaAs $(\lambda=1490 \mathrm{~nm})$. The InP emission intensity decreases towards base of the NP (Fig. 4(b)) while that of InGaAs decreases towards the top of the pillars (Fig. 4(c)), consistent with carrier transfer from the InP NP to the InGaAs layer.

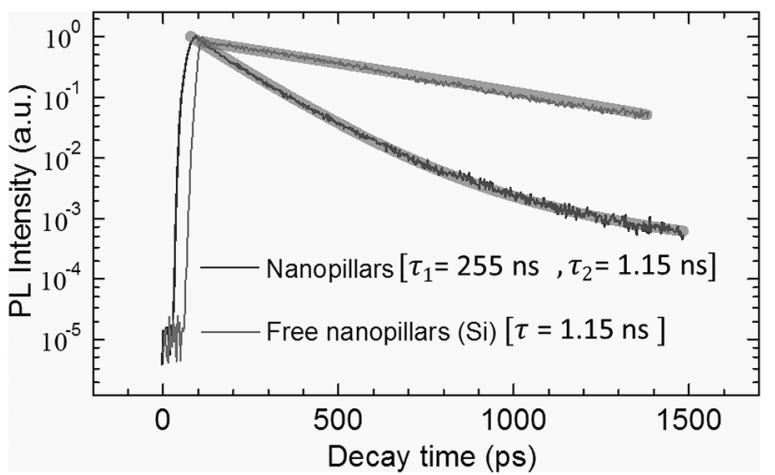

FIG. 3. Room temperature TRPL decay curves measured from InP/InGaAs NPs on InP substrate and free InP NPs transferred on a Si wafer. The exponential fit to the data is represented with thick grey lines. 

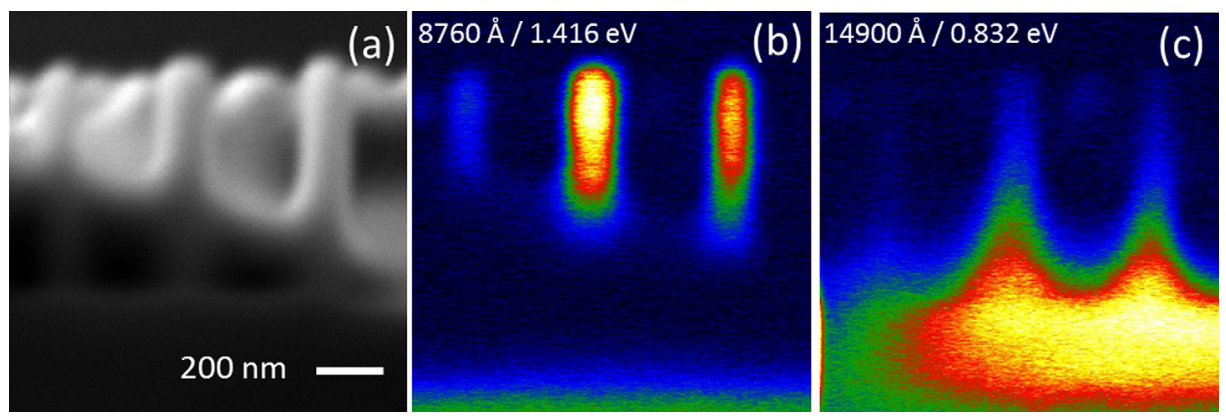

FIG. 4. (a) SEM image of InP NPs with $300 \mathrm{~nm}$ thick InGaAs layer on InP substrate and corresponding monochromatic CL micrographs showing (b) InP emission and (c) InGaAs emission.
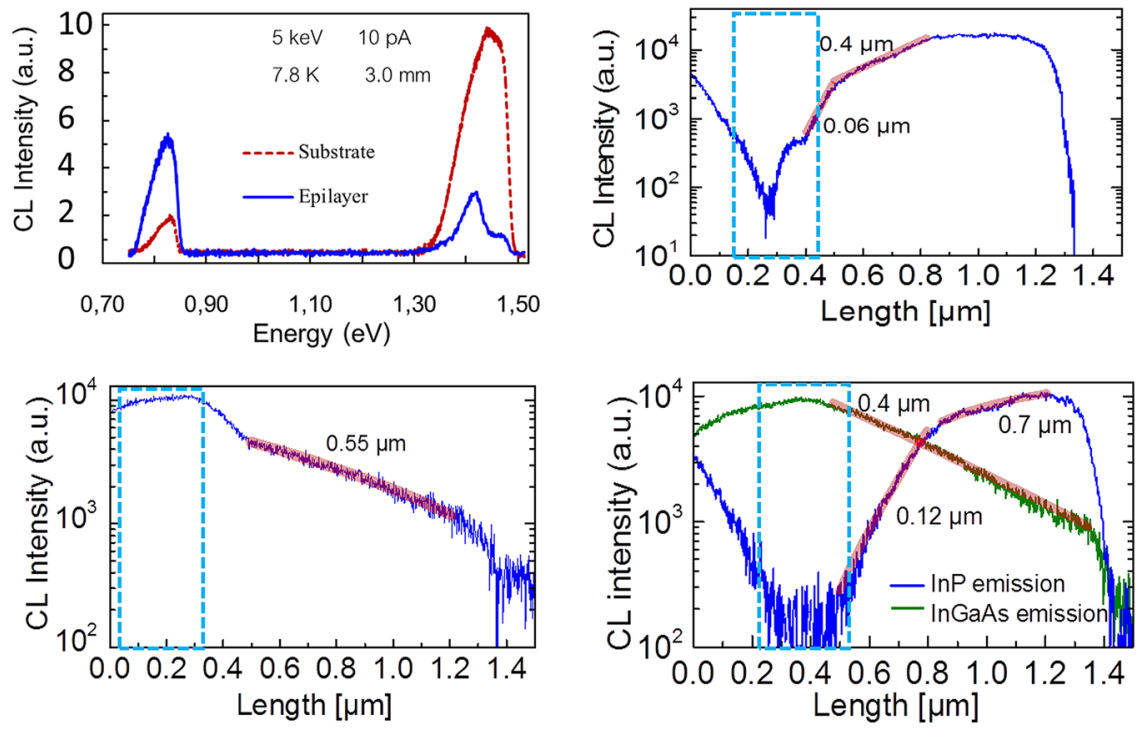

FIG. 5. (a) CL spectra of InP NPs, InGaAs, and n+ InP-substrate. (b) and (c)-Intensity profiles along the NP axis obtained from monochromatic CL images for InP and InGaAs, respectively. The exponential fits used to determine the diffusion lengths are also indicated using the expression $C(X)$ $=C_{o} e^{-X / L_{D}}$. (d) Combined intensity profile obtained from monochromatic CL images for InP and for InGaAs emission. The location of the InGaAs layer is indicated, by a box, in (b)-(d).

Figure 5(a) shows the CL spectra of InP NPs and the InGaAs layer, and the peak positions correspond to their band-edge emission. The line scan, along the NP, obtained from the monochromatic $(\lambda=876 \mathrm{~nm}) \mathrm{CL}$ image, is plotted on Fig. 5(b). The intensity is high at the pillar edge and drops exponentially towards the base. Conversely, the InGaAs intensity obtained from the monochromatic $(\lambda=1490 \mathrm{~nm}) \mathrm{CL}$ image is the highest at the base and decreases exponentially towards the pillar edge. Due to the small diameter of the NPs, we can approximate the diffusion as one-dimensional, and the diffusion length can be determined by fitting a simple exponential to the intensity profile. The carrier diffusion length $\mathrm{L}_{\mathrm{D}}$ as determined by the single exponential fit made to the CL intensity profiles is $\sim 0.5 \mu \mathrm{m}$ in InP (Figs. 5(b)-5(d)). Similar results were obtained from measurements made on several pillars. Here, we note that the change in the slope in CL intensity profiles (Figs. 5(b) $-5(\mathrm{~d})$ ) around the InP NP/InGaAs interface region is due to direct excitation of the InGaAs layer. As the excitation spot gets close to the $\mathrm{InP} / \mathrm{InGaAs}$ interface, a part of the generation volume is located inside the InGaAs layer. In other words, the break in the slope is when we go from pure diffusion in the InP NP to direct excitation of the InGaAs layer. Since the CL spectra of the InP epitaxial layer (peak at $880 \mathrm{~nm}$ ) and InGaAs (peak around $1490 \mathrm{~nm}$ ) are well separated, it is possible to combine line-scan data from the respective monochromatic CL images. Such a combined plot of the intensity profiles along the NP axis (Fig. 5(d)) clearly demonstrates carrier transfer into the InGaAs layer. The InP intensity increases on either side of the InGaAs layer. The determined diffusion lengths are in good agreement with the value calculated from TRPL data. However, a rigorous determination of the diffusion length should include a full three-dimensional analysis.

In conclusion, we have performed a comprehensive analysis of the optical properties of InP NPs fabricated by a top-down approach. We demonstrated that these InP NPs have very good optical quality, comparable to as-grown epitaxial layers. The NPs show high PL intensities, PL linewidths as in as-grown layers even for single NPs, and appreciably long carrier lifetimes $(\sim 1 \mathrm{~ns})$ comparable to bulk values. The determined diffusion lengths in NPs using CL mapping are about $0.5 \mu \mathrm{m}$. The appreciably long diffusion lengths make them attractive for optoelectronic devices including NW/NP solar cells both in axial and radial junction configurations. In addition, CL measurements directly confirm carrier transfer from the InP NPs to the InGaAs layer at their base, and demonstrate the use of a low-band gap layer as a detector to monitor carrier transport in InP NPs. For the specific InP NP/InGaAs structure investigated here, a carrier transfer rate of $\sim 250 \mathrm{ps}$ is determined by TPRL measurements.

The work was performed within the Linné Center for Advanced Optics and Photonics [Grant No.: 349-2007-8664] funded by the Swedish Research Council (VR). Support from the EU network of excellence "Nanophotonics for Energy Efficiency" [Grant No.: 248855] and from "Nanordsun" [Grant No.: 10048] funded by Nordic Innovation centre are also acknowledged. S.N. and N.S. 
acknowledge the Higher Education Commission of Pakistan for partially supporting their PhD studies (scholarship). The CL study was done in the nmC@LU with support from VR and Kurt Alice Wallenberg (KAW) foundation. The authors thank M. Hammar and J. Berggren for the MOVPE growth.

${ }^{1}$ X. Duan, Y. Huang, Y. Cui, J. Wang, and C. M. Lieber, Nature 409, 66 (2001).

${ }^{2}$ M. D. Kelzenberg, S. W. Boettcher, J. A. Petykiewicz, D. B. TurnerEvans, M. C. Putnam, E. L. Warren, J. M. Spurgeon, R. M. Briggs, N. S. Lewis, and H. A. Atwater, Nature Mater. 9, 239 (2010).

${ }^{3}$ S. D. Franceschi, J. A. van Dam, E. P. A. M. Bakkers, L. F. Feiner, L. Gurevich, and L. P. Kouwenhoven, Appl. Phys. Lett. 83, 344 (2003).

${ }^{4}$ C. Thelander, T. Matensson, M. T. Bjork, B. J. Ohlsson, M. W. Larsson, L. R. Wallenberg, and L. Samuelson, Appl. Phys. Lett. 83, 2052 (2003).

${ }^{5}$ F. Wang, P. J. Reece, S. Paiman, Q. Gao, H. H. Tan, and C. Jagadish, Nano Lett. 11, 4149 (2011).

${ }^{6}$ R. Sanatinia, M. Swillo, and S. Anand, Nano Lett. 12, 820 (2012).

${ }^{7}$ M. Law, D. J. Sirbuly, J. C. Johnson, J. Goldberger, R. J. Saykally, and P. Yang, Science 305, 1269 (2004).

${ }^{8}$ Y. Ding, J. Motohisa, B. Hua, S. Hara, and T. Fukui, Nano Lett. 7, 3598 (2007).

${ }^{9}$ L. Cao, P. Fan, A. P. Vasudev, J. S. White, Z. Yu, W. Cai, J. A. Schuller, S. Fan, and M. L. Brongersma, Nano Lett. 10, 439 (2010).

${ }^{10}$ H. Goto, K. Nosaki, K. Tomioka, S. Hara, K. Hiruma, J. Motohisa, and T. Fukui, Appl. Phys. Express 2, 035004 (2009).

${ }^{11}$ L. K. van Vugt, S. J. Veen, E. P. A. M. Bakkers, A. L. Roest, and D. Vanmaekelbergh, J. Am. Chem. Soc. 127, 12357 (2005).

${ }^{12}$ C.-C. Chang, C.-Y. Chi, M. Yao, N. Huang, C.-C. Chen, J. Theiss, A. W. Bushmaker, S. LaLumondiere, T.-W. Yeh, M. L. Povinelli, C. Zhou, P. D. Dapkus, and S. B. Cronin, Nano Lett. 12, 4484 (2012).
${ }^{13}$ L. V. Titova, T. B. Hoang, H. E. Jackson, L. M. Smith, J. M. YarrisonRice, J. L. Lensch, and L. Lauhon, Appl. Phys. Lett. 89, 053119 (2006).

${ }^{14}$ T. B. Hoang, L. V. Titova, J. M. Yarrison-Rice, H. E. Jackson, A. O. Govorov, Y. Kim, H. J. Joyce, H. H. Tan, C. Jagadish, and L. M. Smith, Nano Lett. 7, 588 (2007).

${ }^{15}$ H. C. Casey and E. Buehler, Appl. Phys. Lett. 30, 247 (1977).

${ }^{16}$ L. V. Titova, T. B. Hoang, J. M. Yarrison-Rice, H. E. Jackson, Y. Kim, H. J. Joyce, Q. Gao, H. H. Tan, C. Jagadish, X. Zhang, J. Zou, and L. M. Smith, Nano Lett. 7, 3383 (2007).

${ }^{17}$ H. J. Joyce, J. Wong-Leung, C.-K. Yong, C. J. Docherty, S. Paiman, Q. Gao, H. H. Tan, C. Jagadish, J. Lloyd-Hughes, L. M. Herz, and M. B. Johnston, Nano Lett. 12, 5325 (2012).

${ }^{18}$ R. E. Algra, M. A. Verheijen, M. T. Borgström, L.-F. Feiner, G. Immink, W. J. P. van Enckevort, E. Vlieg, and E. P. A. M. Bakkers, Nature 456, 369 (2008).

${ }^{19}$ P. Caroff, K. A. Dick, J. Johansson, M. E. Messing, K. Deppert, and L. Samuelson, Nat. Nanotechnol. 4, 50 (2009).

${ }^{20}$ S. Naureen, R. Sanatinia, N. Shahid, and S. Anand, Nano Lett. 11, 4805 (2011).

${ }^{21}$ M. Mattila, T. Hakkarainen, H. Lipsanen, H. Jiang, and E. I. Kauppinen, Appl. Phys. Lett. 90, 033101 (2007).

${ }^{22}$ S. Reitzenstein, S. Münch, C. Hofmann, A. Forchel, S. Crankshaw, L. C. Chuang, M. Moewe, and C. Chang-Hasnain, Appl. Phys. Lett. 91, 091103 (2007).

${ }^{23}$ Y. Rosenwaks, Y. Shapira, and D. Huppert, Phys. Rev. B 45, 9108 (1992).

${ }^{24}$ A. Gustafsson, M.-E. Pistol, L. Montelius, and L. Samuelson, J. Appl. Phys. 84, 1715 (1998).

${ }^{25}$ A. Gustafsson, J. Bolinsson, N. Sköld, and L. Samuelson, Appl. Phys. Lett. 97, 072114 (2010).

${ }^{26}$ J. Bolinsson, K. Mergenthaler, L. Samuelson, and A. Gustafsson, J. Cryst. Growth. 315, 138 (2011). 